\title{
Viewpoint
}

\section{Switching a nanomagnet is all in the timing}

\author{
Jonathan Sun \\ IBM Research Division, Thomas J. Watson Research Center, P.O. Box 218, Yorktown Heights, New York 10598
}

Published November 3, 2008

If a magnet is small enough, an electric current carrying polarized spins can flip it around. Scientists are finding clever ways to control this spin-torque effect precisely, both for when it is wanted and when it is not.

Subject Areas: Nanophysics, Spintronics

\author{
A Viewpoint on: \\ Coherent control of nanomagnet dynamics via ultrafast spin torque pulses \\ Samir Garzon, Longfei Ye, Richard A. Webb, Thomas M. Crawford, Mark Covington and Shehzaad Kaka \\ Phys. Rev. B 78, 180401 (2008) - Published November 3, 2008
}

When a spin-polarized current passes through a ferromagnet, it induces a torque on the ferromagnetic moment, an effect referred to as spin-transfer torque, or spin torque for short [1] (see Fig. 1). This ability to flip a magnet with a current directly - rather than indirectly with a magnetic field - opens up a number of technological possibilities for magnetic memory and compact microwave oscillators [2,3]. The spin-torque effect can also be a bit of a nuisance in some devices because it amplifies the thermal noise in a nanomagnet [4-6] - a problem for magnetic read heads. At present, many groups are exploring how to precisely control nanomagnets with a current pulse. Samir Garzon and colleagues at the University of South Carolina, in collaboration with Seagate Research, report [7] they can control the switching of a nanomagnet with a pair of ultrafast, optically generated current pulses better than with the single long current pulses that have been tried in the past. The results, reported in a Physical Review B Rapid Communication, open a new route to nanomagnetic switching.

The spin-torque effect adds a term to the LandauLifshitz-Gilbert (LLG) equation of motion for a magnetic moment [8]. The new term describes the spin torque and is proportional to $\left(\mathbf{n}_{s} \times \mathbf{m}\right) \times \mathbf{m}$, where $\mathbf{m}$ is the magnetic moment of the ferromagnet and the spins in the current are polarized along $\mathbf{n}_{s}[9]$. The direction of the spin torque turns out to be aligned with the damping torque, a typically small dissipative force that, given enough time, brings a magnetic moment back to its easy axis (the energetically most favorable direction in which the moment can lie). The spin-torque effect can therefore either increase or decrease the natural damping of the nanomagnet. If the spin torque is sufficiently large in the direction opposite to the natural damping, the magnetic moment could become unstable as it enters an amplified precession and eventually reverses its direction.
Spin torque is usually only directly observable when the ferromagnet is less than about $100 \mathrm{~nm}$ across [2], but as many modern electronics components are shrinking below this scale, the effect can become quite important. Because the direction of the spin torque is proportional to $\left(\mathbf{n}_{s} \times \mathbf{m}\right) \times \mathbf{m}$, it is zero when $\mathbf{m}$ and $\mathbf{n}_{s}$ are parallel, and any spin-torque effect in a system where the spins in the current are perfectly aligned with the easy axis would, in principle, take a long time to initiate $[4,10,11]$. In reality, the initial angle of the nanomagnet is thermally distributed about the easy axis, and this introduces some uncertainty in the initial direction of the spin torque. It has been shown that a noncollinear initial state can improve the shot-to-shot repeatability of a spin-torque-induced switch [12]. In such an arrangement, the magnetic easy axis is at a finite angle with the spin polarization of the current, which reduces uncertainties in the initial conditions and facilitates a well-defined initial spin-torque direction. Unfortunately, when there is a finite angle between the magnetic easy axis and $\mathbf{n}_{s}$, the spin torque changes sign with respect to the damping torque within a single precession cycle once the orbit is within a cone angle that is less than the angle between $\mathbf{m}$ and $\mathbf{n}_{S}$ (see Fig. 1). This increases the net average threshold current for spintorque-induced switching. This angular dependent effect has been quantitatively predicted [13] and observed for current thresholds over a time scale that is long compared to the natural precession frequency of the nanomagnet [14].

The precession frequency of nanomagnets is usually on the order of a few to a few tens of GHz. Experiments performed in the time domain can measure the switching probability distribution and determine the precession dynamics. In combination with numerical simulations, these studies reveal the dynamics and the effect of an offset bias field (that cants the easy axis away from $\mathbf{n}_{s}$ ) on the precession [15]. A direct, controlled observa- 


\section{Phys̄īcs}

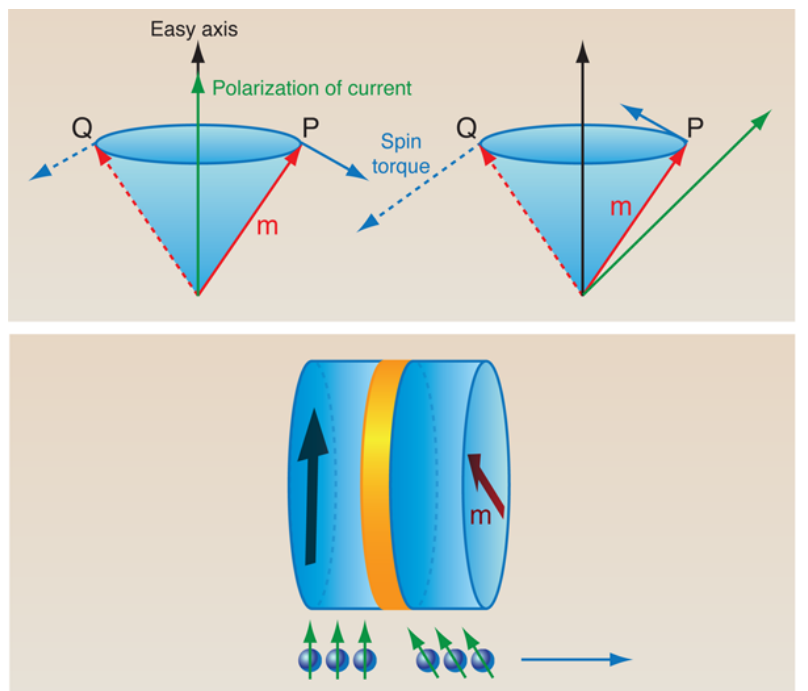

FIG. 1: The spin-torque effect in a nanomagnet. (top left) If the polarization of the spins in the current $\left(\mathbf{n}_{s}\right.$, green arrow) is parallel to the easy axis (black arrow), the spin torque opposes the natural damping and opens up the angle of the precession cone at all points along the cone. (top right) If $\mathbf{n}_{s}$ makes a finite angle with the easy axis, the spin torque opens the precession cone angle around a half circle centered at point $Q$, but closes the cone angle in the other half circle centered at point $P$. Applying two successive spin-torque pulses either both at point $Q$, or at points $P$ and $Q$, increases or decreases the net effectiveness of the spin torque in inducing a dynamic switch. (bottom) Schematic of the nanopillar structure. The free layer is the right layer and the current is applied along the horizontal axis of the pillar. The polarization of the current (green arrows) also rotates as a result of the spin-torque effect. (Illustration: Alan Stonebraker/stonebrakerdesignworks.com)

tion of the coherent precession for such a noncollinear spin-torque effect however, would require a time resolution well below a nanosecond.

All of this sets the stage for the work of Garzon et al. The group devised an ingenious approach to demonstrate the coherent control of spin-torque dynamics in a magnetic nanopillar. The sandwich-like structure, shown in Fig. 1, consists of a CoFe ferromagnetic layer that has a fixed magnetization, a thin nonmagnetic $\mathrm{Cu}$ spacer, and a CoFe ferromagnetic layer with a magnetization that is free to rotate. The whole device is 75 $\mathrm{nm} \times 150 \mathrm{~nm}$ across. They control the rotation of the free layer by creating a pair of pulsed currents with very narrow pulse widths (58 ps or less) and with a precise and tunable delay between the pulses that could be extended to over a nanosecond. They used a mode-locked, pulsed laser with the appropriate beam split, delay, and recombination to create a pair of optical pulses that are then converted to electrical pulses with a photoconductive switch. They then successfully coupled these pulse currents into the nanopillar for the experiment.

The final resting position of the nanomagnet is detected by measuring the dc resistance of the sandwich structure (the resistance is several percent less when the free and fixed layers are parallel as opposed to antiparallel). Since the pulse width of about 50 ps is much shorter than the natural precession period of the nanomagnet, the pulsed current acts like an instantaneous impulse excitation. When the pulse has large enough amplitude, it alone is enough to pump a sufficient amount of spin angular momentum into the nanomagnet, causing it to reverse its direction. What is more intriguing is the demonstration here that one could use two pulses, timed precisely to arrive at different points on the precession trajectory of the free layer of the nanomagnet. This allows one to either coherently add to or subtract from the nanomagnet a controlled amount of spin angular momentum, affecting the subsequent precession. The proof that the pulses contribute coherently is that the switching probability oscillates with the delay between the pulses. The group also estimates that the coherence time of the nanomagnet precession dynamics is of order $1 \mathrm{~ns}$, and perhaps is limited by magnetic damping.

This experiment provides an excellent demonstration of the nature of the dynamics involved in a spin-torque switch. It is also an effective way to probe the process of decoherence in real-life spin-torque devices. Understanding decoherence is important for the optimization of the threshold current and switching speed of the device, and the methodology developed by this work is a valuable addition to the experimental exploration of spin-torque effects in many materials and device systems.

\section{References}

[1] For a recent review, see D. C. Ralph and M. D. Stiles, J. Magn. Magn. Mater. 320, 1190 (2008).

[2] J. Z. Sun, IBM J. Res. Dev. 50, 81 (2006).

[3] M. Hosomi, H. Yamagishi, T. Yamamoto, K. Bessho, Y. Higo, K. Yamane, H. Yamada, M. Shoji, H. Hachino, C. Fukumoto, H. Nagao, and H. Kano, IEDM Technical Digest, IEEE International, 5-7 Dec. Pages 459-462, (2005).

[4] R. H. Koch, J. A. Katine, and J. Z. Sun, Phys. Rev. Lett. 92, 088302 (2004); J. Z. Sun, T. S. Kuan, J. A. Katine, and R. H. Koch, Proc. SPIE, 5359, 445 (2004).

[5] Z. Li and S. Zhang, Phys. Rev. B 69, 134416 (2004); D. M. Apalkov P. B. Visscher, Phys. Rev. B 72, 180405 (2005).

[6] J.-G. Zhu, N. Kim, Y. Zhou, Y. Zheng, J. Chang, K. Ju, X. Zhu, and R. M. White, J. Appl. Phys. 40, 2323 (2004).

[7] S. Garzon, L. Ye, R. A. Webb, T. M. Crawford, M. Covington, and S. Kaka, Phys. Rev. B 78, 180401 (2008).

[8] E. M. Lifshitz and L. P. Pitaevskii, Statistical Physics, Part II, Chapter 7, Magnetism. Wheaton \& Co. Ltd. Exeter, p. 285 (1981).

[9] See, for example, D. V. Berkov and J. Miltat, J. Magn. Magn. Mater. 320, 1238 (2008) and references therein.

[10] J. Z. Sun, Phys. Rev. B 62, 570 (2000).

[11] A. A. Tulapurkar, T. Devolder, K. Yagami, A. Fukushima, and Y. Suzuki, Appl. Phys. Lett., 85, 5358.

[12] I. N. Krivorotov, N. C. Emley, J. C. Sankey, S. I. Kiselev, D. C. Ralph, and R. A. Buhrman, Science 307, 228 (2005).

[13] J. Z. Sun, J. Magn. Magn. Mater. 202, 157 (1999). 
[14] F. B. Mancoff, R. W. Dave, N. D. Rizzo, T. C. Eschrich, B. N. Engel, and S. Tehrani, Appl. Phys. Lett. 83, 1596 (2003).

[15] T. Devolder, C. Chappert, and K. Ito, Phys. Rev. B 75, 224430
(2007); T. Devolder, J. Hayakawa, K. Ito, H. Takahasi, S. Ikeda, J. A. Katine, J. Appl. Phys. 103, 07A723 (2008).

\section{About the Author}

\section{Jonathan Sun}

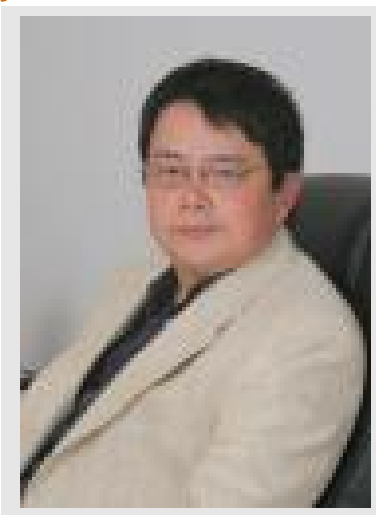

Jonathan Sun received a B.S. degree in physics from Fudan University, Shanghai, China, in 1984, and M.S. and Ph.D. degrees in applied physics from Stanford University in 1986 and 1989, respectively. For the following two years, he worked at Superconductor Technologies Inc. in Santa Barbara, California, as a member of the technical staff. He subsequently joined IBM at the Thomas J. Watson Research Center as a Research Staff Member in the Physical Sciences Department where he has worked on advanced superconducting and magnetic devices. Dr. Sun is a fellow of the American Physical Society. 